\title{
Effect of Truncation on the Performance of Busemann Inlet
}

\author{
Zhi Zhao \& Wenyan Song \\ School of Power and Energy \\ Northwestern Polytechnical University \\ Xi' an 710072, China \\ E-mail: zhaozhinwpu@gmail.com
}

\begin{abstract}
The aerodynamic design of hypersonic inlets is a critical issue for the overall performance of an air-breathing propulsion system. The design procedure of basic Busemann inlet is presented. Five truncated Busemann inlets with a contraction ratio of 9 are designed for Mach 6 . These inlets have different truncation angles range from $0^{\circ}$ to $6^{\circ}$. Numerical simulations have been performed to investigate the effects of truncation on inlet performance. The results of numerical simulations confirmed the good performance of the Busemann inlet. The truncation angle of $4^{\circ}$ is preferred.
\end{abstract}

Keywords: Hypersonic inlet, Truncation, Busemann inlet, Contraction ratio

\section{Introduction}

The aerodynamic design of hypersonic inlets is a critical issue for the overall performance of an air-breathing propulsion system. The inlet of a scramjet engine serves to capture and compress the engine air flow. The inlet must compress the flow with minimal skin friction, heat transfer, and shock loss, while providing uniform flow to the combustor. During the past, many types of hypersonic inlets, such as two-dimensional inlets, axisymmetric inlets and sidewall-compression inlets, have been evaluated (Billig, F. S., 2003).

Recently there has been a renaissance of the inward turning inlets for hypersonic air-breathing propulsion (Adam Siebenhaar ,2006;Bulman, M.J.,2006). The two-dimensional inlets compress the flow only in one direction, while the inward turning inlets have three-dimensional compression surface. Inward turning inlets derived from efficient compression flowfield offer unique performance. The potential benefits include improved flowpath performance, greater structural efficiency, and great flexibility in vehicle configuration development. Due to the highly integrated nature of hypersonic vehicles, the flowpath configuration strongly influences the overall vehicle configuration.

Previous work has shown that axisymmetric Busemann and streamtraced Busemann inlets are good candidates for hypersonic air-breathing vehicles. Billig (Billig, F. S., 1995) described the scramjet missile concept at APL/JHU between 1962 and 1978, which was based on tracing streamlines through a Busemann flowfield. Van Wie (Van Wie, D M, 1992) and Billig (Billig, F. S., 1999) presented the sample Busemann inlet design.

\section{Busemann Inlet Design Procedure}

As showed in Figure 1, the Busemann inlet is an axisymmetric, internal contraction inlet which has very low inviscid losses as well as a small ratio of flow cross-section area to wetted surface area. The Busemann inlet flowfield is an inviscid inward turning axisymmetric flowfield that terminates in a conical shock wave. At the design Mach number, the compression upstream of the conical shock wave is isentropic and the flow down -stream is uniform. The conical shock wave is the only entropy producing feature of inviscid design. The resulting inviscid compression efficiency is remarkably higher than other classes of inlets. This type of flowfield is well suited for scramjet engine inlets.

The flowfield of the Busemann inlet between the freestream and the conical shock wave is conical symmetric and irrotational. So the Taylor-Maccoll equation for the external flowfield past a cone can be used here. In spherical coordinate system, the Taylor-Maccoll equation for a calorically perfect gas can be written as follows:

$$
\begin{gathered}
v_{\theta}\left(v_{r} \frac{d v_{r}}{d \theta}+v_{\theta} \frac{d v_{\theta}}{d \theta}\right)-a^{2}\left(\frac{d v_{\theta}}{d \theta}+2 v_{r}+v_{\theta} \cot \theta\right)=0 \\
v_{\theta}-\frac{d v_{r}}{d \theta}=0
\end{gathered}
$$


The solution of equation (1) and (2) is started from the downstream shock, because of a singularity at the upstream Mach wave. In the numerical solution, the post shock Mach number $\mathrm{M}_{3}$ and the shock angle $\theta_{\mathrm{s}}$ are specified. The parameters upstream the conical shock wave can be solved from relations across oblique shock wave. Then the flowfield inside the inlet can be calculated. The Taylor-Maccoll equation is then numerically integrated using the Runge-Kutta algorithms. Numerical integration is then performed in the upstream direction until the freestream condition is reached. Also, the streamline integration can be terminated at some angle, formed by the freestream vector and the velocity vector, which is called truncation angle.

\section{Effects of Truncation Angle}

The relative large length and corresponding heavy weight of the basic Busemann inlets prevent it from realistic application. An efficient approach is truncation of the leading edge (Billig, F. S., 1999). Therefore in this study, the effects of truncation angle on inlet performance were examined. Numerical simulations have been performed to evaluate the performance of these inlets. The effect of the truncation angle on kinetic energy efficiency, compressive efficiency and Mach number are analyzed. The viscous effects and the heat loss through the wall are taken into account.

Computational simulations for the axisymmetric inlets for both inviscid and viscous analysis were performed. The Reynolds averaged Navier-Stokes Equations were solved using the CFD code. This code utilizes finite volume methods. The Roe's second order flux difference splitting algorithm was used. The k- $\varepsilon$ turbulence model with wall function was adopted. The structured grids had 600 grids axially, 200 points radically and were clustered near the wall to fulfill $\mathrm{y}^{+} \approx 10$. The freestream conditions correspond to flight Mach number 6 , altitude of $25 \mathrm{~km}$. A wall temperature of $1000 \mathrm{k}$ was set for the isothermal cases.

A more equitable comparison should be to examine truncated inlets with same contraction ratio. Five truncated Busemann inlets with a contraction ratio of 9 are designed for Mach 6. Figure 2 shows geometry profiles of these inlets, and Mach number contours of inviscid flowfields. Because of symmetry, only half of the flowfield is displayed. These inlets have different truncation angles that range from $0^{\circ}$ to $6^{\circ}$. Each inlet was generated in an iterative manner by holding the freestream Mach number and capture height constant while varying the throat Mach number, until a specified contraction ratio was obtained. For example, the Busemann inlet of with a contraction ratio of 10.6 at Mach 6 was truncated at $4^{\circ}$, resulting the $\mathrm{CR}=6$, to reduce the size and weight of the inlet.

Figure 2 also shows that the conical shock wave are nearly perfectly cancelled at the shoulder for truncation angle less than $4^{\circ}$.

Figure 3 shows the length of inlet with different truncation angles, the length are normalized by the exit radius. As the truncation angle increases, the length of inlet decreases rapidly, by as much as $35 \%$ for the $4^{\circ}$ truncation and $45 \%$ for the $6^{\circ}$ truncation.

The effect of the truncation angle on exit pressure and Mach number is shown in figure 4. For inviscid case the exit pressure increases with the truncation angle. As the truncation angle is increased, an oblique shock is formed at the sharp leading edge. When viscous effects are included, the exit pressure is in the range of $85 \sim 90 \mathrm{kPa}$, and the lowest exit pressure is reached at $3^{\circ}$ inlet. The inviscid exit Mach number decrease slightly with the increasing truncation angle. For viscous cases, the Mach number is near 2.8 .

Figure 5 shows the effect of the truncation angle on compression efficiency and kinetic energy efficiency. The highest kinetic energy efficiency is reached by the $0^{\circ}$ inlet. This near perfect efficiency is a result of the inviscid flowfield having an ideal isentropic compression followed by a single conical shock. As the truncation angle is increased, the shock wave from the leading edge introduces a second source of total pressure loss, and drives down the kinetic energy efficiency. When viscous effects are included, the adiabatic kinetic energy efficiency shows a decrease of approximately $1.8 \%$ for all truncation angles. An additional $1.0 \%$ decrease occurs when heat loss to the wall $\left(\mathrm{T}_{\mathrm{w}}=\right.$ $1000 \mathrm{~K}$ ) is taken into account.

For the compression efficiency, it can be seen that the inviscid $0^{\circ}$ inlet performs perfectly, while the larger truncation angles show a substantial loss in efficiency. When viscous effects are included, the compressive efficiencies remain constant near $96 \%$ for less than $4^{\circ}$.

Based on the trends shown in Fig. 3, 4, and 5, we have concluded that a truncation angle between $2^{\circ}$ and $4^{\circ}$ gives a good balance of inlet performance. The $2^{\circ}$ inlet and $4^{\circ}$ inlet are approximately $25 \%$ and $35 \%$ shorter than the $0^{\circ}$ inlet. The truncation angle of $4^{\circ}$ is preferred.

\section{Conclusions}

The results of numerical simulations confirmed the high performance of these inlets.As the truncation angle is increased, an oblique shock is formed at the sharp leading edge. And that shock distorts the flowfield, reduces the total pressure, and decreases the inlet's efficiency. The results show that the best truncation angle is $4^{\circ}$.Compared with basic Busemann inlet, the length of the $4^{\circ}$ truncated inlet decrease about $35 \%$, while performance still relative good. 


\section{References}

Adam Siebenhaar, Bogar T.J. (2006). The Impact of Round Combustors on TBCC Propulsion and Hypersonic Cruise Vehicles, AIAA 2006-7986.

Billig, F. S. (1995).Supersonic Combustion Ramjet Missile, Journal of Propulsion and Power, 11, pp: 1139-1146.

Billig, F. S., Baurle, R., and Tam, C. (1999). Design and Analysis of Streamline Traced Hypersonic Inlets, AIAA 99-4974.

Billig, F. S., Jacobsen, L. (2003). Comparison of Planar and Axisymmetric Flowpaths for Hydrogen Fueled Space Access Vehicles, AIAA 2003-4407.

Bulman, M.J., Adam Siebenhaar (2006). The Rebirth of Round Hypersonic Propulsion, AIAA 2006-5035.

Van Wie, D M, Molder, S. (1992). Application of Busemann Inlet Designs for Flight at Hypersonic Speeds, AIAA 92-1210.

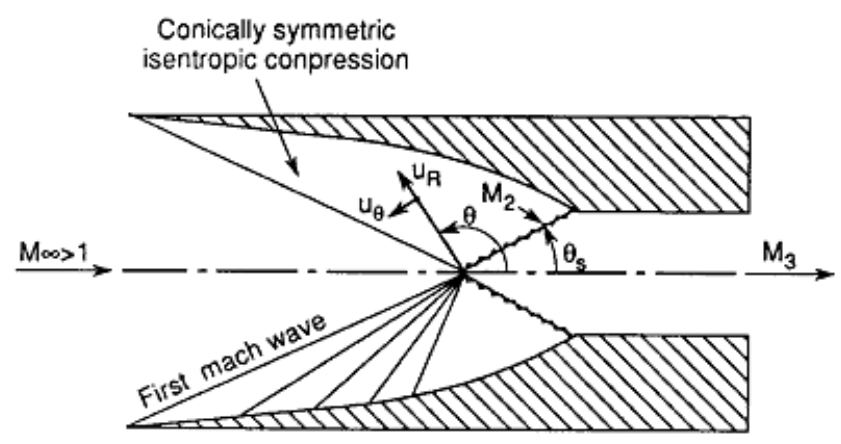

Figure 1. Flowfield of the Busemann Inlet (Van Wie, D M,1992)

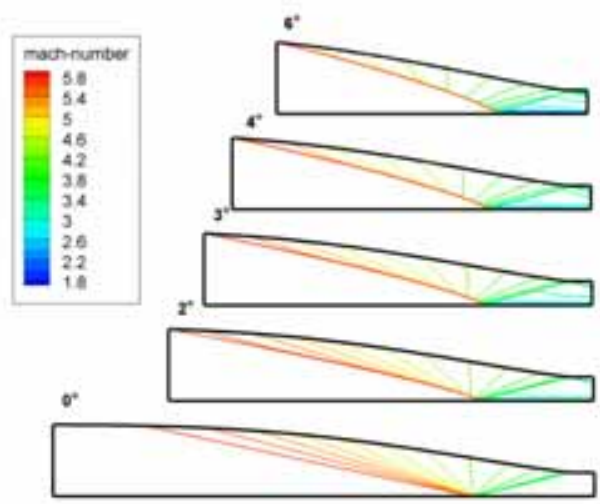

Figure 2. Busemann Inlet flowfield with Different Truncation Angles 


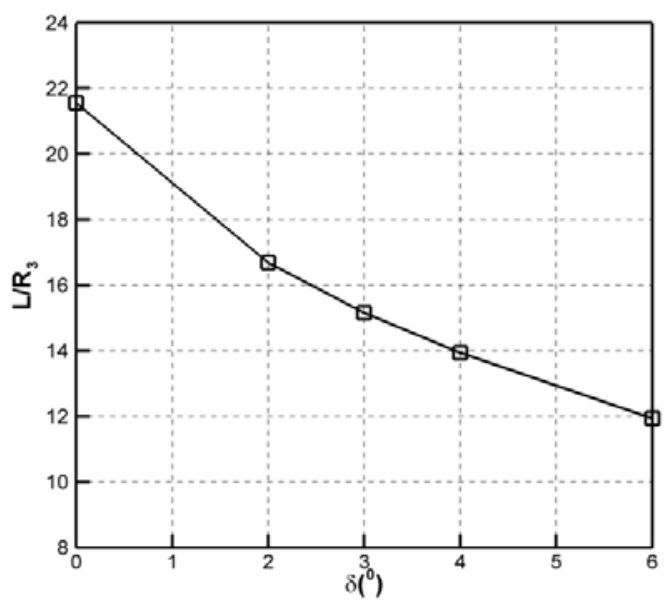

Figure 3. Length of Inlet with Different Truncate Angles

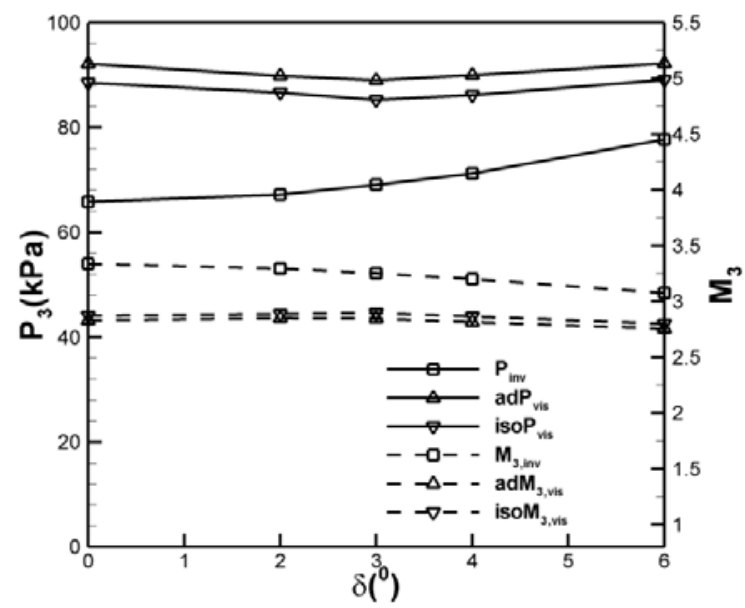

Figure 4. Effect of Truncation angle on pressure and Mach Number

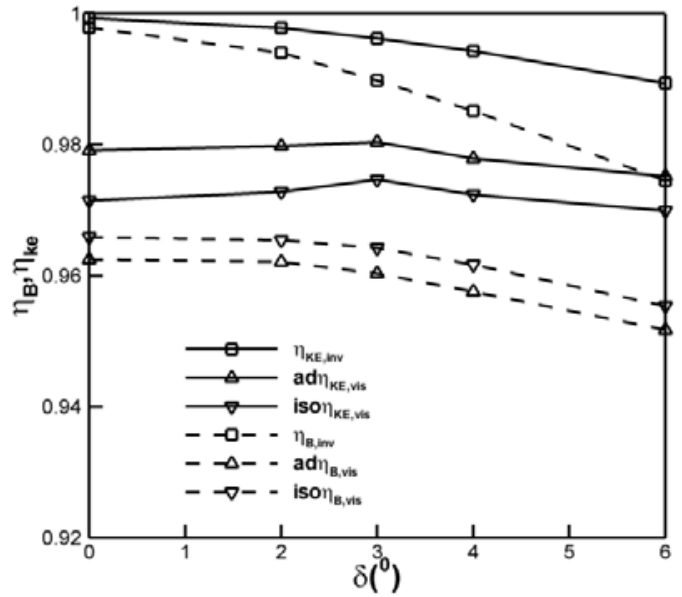

Figure 5. Effect of Truncation angle on compression efficiency and kinetic energy efficiency 